\title{
The anti-phytoalexin gene Bx-cathepsin W supports the survival of Bursaphelenchus xylophilus under Pinus massoniana phytoalexin stress
}

\author{
Feng Wang ${ }^{\dagger}$, Qiaoli Chen ${ }^{\dagger}$, Ruizhi Zhang, Danlei Li, Yaming Ling and Ruiqing Song ${ }^{*}$ (D)
}

\begin{abstract}
Background: Pine trees challenged by Bursaphelenchus xylophilus invasion produce phytoalexins to combat this nematode. Nevertheless, the phytoalexins of Asian pine trees are ineffective against B. xylophilus. The anti-phytoalexin genes of $B$. xylophilus disable almost all Asian pine phytoalexins, which has allowed B. xylophilus to devastate pine forests in eastern Asia over the last four decades. However, to date, the factors that stimulate anti-phytoalexin gene expression and the mechanisms by which these genes act are not well understood.

Results: Here, we described anti-phytoalexin genes in B. xylophilus using transcriptomic and bioinformatics analyses. The genes that were induced by both Pinus massoniana and carvone and had similarly elevated expression trends were considered anti-phytoalexin genes. Altogether, 187 anti-phytoalexin genes were identified, including 4 cathepsin genes. KEGG pathway enrichment indicated that those cathepsins were related to the Lysosome pathway. Since cathepsins help to maintain metabolic homeostasis by participating in the degradation of heterophagic and autophagic material, the lysosomal cathepsin gene Bx-cathepsin $W$ was cloned and characterized. The results of the RNAi assessment indicated that the knockdown of Bx-cathepsin $W$ reduced the survival rates of $B$. xylophilus under carvone or P. massoniana stress. The correlation between $B x$-cathepsin $W$ and the susceptibility of pines showed that $B x$-cathepsin W might help improve the anti-phytotoxin ability of B. xylophilus.
\end{abstract}

Conclusions: The results indicated that the anti-phytoalexin gene Bx-cathepsin W supported the survival of B. xylophilus under $P$. massoniana phytoalexin stress. The cDNA library sequencing, differentially expressed gene identification, and WGCNA algorithm analysis provided insight at a systemic level into the gene regulation of $B$. xylophilus in response to the immune reaction of $P$. massoniana. These results will lead to a better understanding of the function of nematode defenses in host innate immunity.

Keywords: Phytoalexin, Carvone, Bursaphelenchus xylophilus, Bx-cathepsin W, WGCNA

\section{Background}

The pine wood nematode Bursaphelenchus xylophilus, the causative agent of a devastating epidemic of pine wilt disease, is a migratory endoparasite that causes severe damage to pine forests in East Asia and Europe [1, 2]. In both the nematode's native habitat in North America and in new epidemic areas in East Asia and Europe, B. xylophilus has

\footnotetext{
* Correspondence: RuiqingSongnefu@163.com

${ }^{\dagger}$ Feng Wang and Qiaoli Chen contributed equally to this work.

Key Laboratory of Sustainable Forest Ecosystem Management-Ministry of Education, School of Forestry, Northeast Forestry University, Harbin 150040, Heilongjiang, People's Republic of China
}

evolved tolerance to pine defenses. Especially in China [3] and Japan, the nematode can completely defuse all natural host resistance.

Susceptible host plants are not completely vulnerable because their immune system can combat the vast majority of attackers [4]. Resistance to disease can be described on several levels to stop pathogens from developing in host tissues after infection based on physical and biochemical factors [5]. One type of biochemical response that is strongly associated with defense is the accumulation of phytoalexins, which are defined as low-molecular-

(c) The Author(s). 2019 Open Access This article is distributed under the terms of the Creative Commons Attribution 4.0 International License (http://creativecommons.org/licenses/by/4.0/), which permits unrestricted use, distribution, and reproduction in any medium, provided you give appropriate credit to the original author(s) and the source, provide a link to the Creative Commons license, and indicate if changes were made. The Creative Commons Public Domain Dedication waiver (http://creativecommons.org/publicdomain/zero/1.0/) applies to the data made available in this article, unless otherwise stated. 
weight antimicrobial compounds that are produced after infection [6]. Phytoalexins, which are chemically diverse, include simple phenylpropanoid derivatives, flavonoidand isoflavonoid-derived phytoalexins, sesquiterpenes, polyketides, etc. [5]. The production of several phytoalexins in Pinus strobus after the infection of B. xylophilus $[7,8]$ suggests that the host-pathogen interaction is involved in triggering phytoalexin biosynthesis. However, it is indisputable that B. xylophilus can withstand phytoalexins and survive. We believe that some antiphytoalexin genes regulate nematode resistance to phytoalexins. Genomics research has identified many B. xylophilus stress response-related genes [9]. However, whether all of these genes are anti-phytoalexins requires further study. Thus, in this study, we investigated the anti-phytoalexin gene transcript patterns by constructing and sequencing 4 cDNA libraries from $B$. xylophilus. We were particularly interested in determining whether $B x$-cathepsin $W$ functions in the anti-phytoalexin process of $B$. xylophilus in response to the stress of the pine host of $P$. massoniana and the botanical nematicide carvone. This study presents our investigations into the mechanism of $B$. xylophilus against phytoalexin and the molecular characterization of a $B x-c a-$ thepsin $W$ gene.

\section{Methods}

Induction of $B$. xylophilus gene expression by $P$. massoniana

B. xylophilus nematodes (collected in GZ, China) were cultured on Botrytis cinerea at $25^{\circ} \mathrm{C}$ in the dark. A Baermann funnel was used to extract the nematodes (male, female, and juvenile mixed together at a ratio of 1:1:2). To characterize the B. xylophilus gene transcript patterns induced by the host $P$. massoniana, a nematode

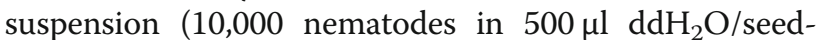
ling) was inoculated into 3-year-old P. massoniana seedlings. The suspension was pipetted into an Eppendorf tube, which was filled with degreasing cotton, and inoculated into an artificial wound ( $1 \mathrm{~cm}$ long by $0.5 \mathrm{~cm}$ wide) on the seedling. The wound was made by cutting and peeling back the bark to expose the xylem [10]. Three days after inoculation, nematodes were isolated from $P$. massoniana seedlings and then frozen in a mortar with liquid nitrogen and powdered using a pestle for RNA extraction and transcriptome sequencing (set as Treatment $1)$. An equal number of nematodes cultured on $B$. cinerea, set as a control (CK1), were also powdered.

\section{Induction of $B$. xylophilus gene expression by Carvone}

An assay was performed to characterize the gene transcript patterns of the B. xylophilus genes induced by carvone, set as Treatment 2. Carvone, an effective inhibitor of $B$. xylophilus motility, was isolated from the essential oils of $P$. sylvestris in a preliminary trial. Stock solutions of carvone were prepared by dilution with $70 \%$ ethanol. Working solutions were obtained by diluting the stock solutions with distilled water containing the polysorbate surfactant 20 (Tween-20) [11]. Treatment 2 was performed in the dark at $25^{\circ} \mathrm{C}$ in 24 -well polystyrene culture plates containing $500 \mu \mathrm{l}$ carvone $(1600 \mathrm{mg} / \mathrm{L})$ per well or $\mathrm{ddH}_{2} \mathrm{O}$ containing ethanol $(0.8 \% \mathrm{v} / \mathrm{v})$ and Tween-20 (0.3\% v/v) as CK2. Nematodes (10,000 nema-

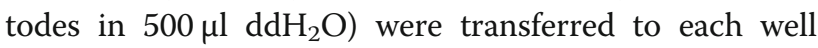
and treated for 1 day. Nematodes in Treatment 2 or CK2 were then collected and frozen in a mortar with liquid nitrogen and powdered for RNA extraction and transcriptome sequencing.

\section{Transcriptome sequencing and differentially expressed gene identification}

Total RNA from treated B. xylophilus (Treatment 1 and Treatment 2) and CKs (CK1 and CK2) was extracted from the nematode powder using TRIzol (Invitrogen, Carlsbad, CA, USA) and then cleaned using an RNeasy Minikit col-

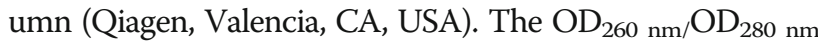
and $\mathrm{OD}_{260 \mathrm{~nm}} / \mathrm{OD}_{230 \mathrm{~nm}}$ values of the extracted RNA were quantified using a NanoDrop (Thermo Scientific, Wilmington, DE, U.S.A.). Then, 4 cDNA libraries representing Treatment 1, CK1, Treatment 2, and CK2 were prepared [12] and sequenced by BGI-Shenzhen (Shenzhen, Guangdong, China) according to the BGISEQ-500 standard protocol (Additional file 1: Text S1 and S2) [13, 14]. To analyze the differential expression profiles, 3 biological replicates were performed. The assembly of the clean reads and the quality of the assembly were critically assessed by BGIShenzhen (Shenzhen, Guangdong, China) before subsequent analysis (Additional file 1: Text S3). Clean reads were mapped to the reference genome of B. xylophilus (BioSample: SAMEA2272519), which is available at NCBI (http:// www.ncbi.nlm.nih.gov/assembly/310678), using HISAT2 (v2.0.4, http://www.ccb.jhu.edu/software/hisat) [15]. The dataset was deposited in the Sequence Read Archive (SRA).

To identify the differentially expressed B. xylophilus genes, we normalized (by the mean of transcripts per million clean reads, TPM) the read distribution for the gene expression levels in each library to construct an effective library size. The differential gene expression was analyzed according to the method of Li (Additional file 1: Text S4) [16]. The differential gene expression levels were compared using the $\log _{2}$ (Treatment $1 / \mathrm{CK} 1$ ) or $\log _{2}$ (Treatment $2 / \mathrm{CK} 2$ ) fold changes of the normalized reads. After the dispersion of each gene was estimated, differentially expressed genes were identified by DESeq using adjusted false discovery rate (FDR) [17]. The threshold of differentially expressed genes was set to $\log _{2}$ fold change $>1$ or $<-1 \quad($ FDR $<0.05)$. The threshold of significant differentially expressed genes was set to $\log _{2}$ fold change $>1$ or $<-1($ FDR $<0.01)$. 


\section{Identification of anti-phytoalexin genes}

The differentially expressed B. xylophilus genes induced by the host $P$. massoniana and the botanical nematicide carvone were designated $P$. massoniana-induced genes and carvone-induced genes, respectively.

The genes that were induced by both $P$. massoniana and carvone and had similarly elevated expression trends were considered anti-phytoalexin genes. Since B. xylophilus must face not only phytoalexin but also a more complex mixture of compounds in the resin canals of its pine hosts, carvone-induced B. xylophilus genes were used to filter $P$. massoniana-induced genes to obtain anti-phytoalexin genes. The union of the promoted $P$. massoniana-induced genes and promoted carvoneinduced genes were designated as the anti-phytoalexin genes in this study. Kyoto Encyclopedia of Genes and Genomes (KEGG) analysis and enrichment were performed for the anti-phytoalexin genes.

\section{Bx-cathepsin W cloning and Bx-cathepsin W modeling}

Total RNA was extracted from the powder of Treatment 2 nematodes by using TRIzol (Invitrogen, Carlsbad, CA, USA), and the AMV reverse transcription system (Promega, Madison, WI, USA) was subsequently applied to obtain the first-strand cDNA. Bx-cathepsin $W$ was then cloned using the first-strand $\mathrm{cDNA}$ as the template with the primer pair 5' GCC TCC GAT CAT GCG ACT AT 3' and Oligo $(\mathrm{dT})_{18}$. The deduced amino acid sequence of Bx-cathepsin W was submitted to the SWISSModel server [18] for comparative protein structure modeling. Threedimensional structure alignments of the predicted $\mathrm{Bx}$ cathepsin W were prepared with the program PyMOL [19].

\section{RNAi of Bx-cathepsin W}

Fluorescent siRNAs corresponding to Bx-cathepsin $W$ (siRNA sequence: 5' GGG UGG GCC GUC GAC CAC GGC GUU CUG AC [dT] [dT] 3', labeled with 5carboxyfluorescein (FAM) and named Bx-cathepsin $W$ RNAi), or Bx-Actin (siRNA sequence: 5' GCC UUC UUU CUU GGG UAU GGA AUC UGC CG [dT] [dT] 3', labeled with cyanine dyes 3 (Cy3) and named Bx-Actin RNAi) were constructed separately. As a nontargeting RNAi treatment, a nontargeting siRNA (siRNA sequence: 5' AGG AGC UGU UCA CCG GGG UGG UGC CCA UCC U [dT] [dT] 3') was selected.

RNAi was performed using mixed stages of nematodes soaked in M9 buffer with $10 \mathrm{mM}$ octopamine and siRNAs $(2 \mu \mathrm{g} / \mu \mathrm{l})$ or M9 buffer with $10 \mathrm{mM}$ octopamine as a control overnight in the dark at $25^{\circ} \mathrm{C}$, as outlined in Wang, et al. [3]. The RNAi-treated and control nematodes were divided into 3 groups. The first group was used to determine the extent of RNAi by real-time quantitative PCR (RT-qPCR) and photographed under a fluorescence microscope (Olympus Bx51, Japan). RT-
qPCR was performed with a GoTaq 2-Step RT-qPCR System Kit (Promega, Madison, WI, USA) and a Stratagene Mx3000P qPCR system (Agilent, Santa Clara, CA, USA) to validate the transcript levels of Bx-cathepsin $W$ with the primers BxCWqF (TTG CAT TCT ACG GCC AGT CC) and BxCWqR (ACT GAC TTT CGA TGG CTC CG). Bx-Actin RNA was used as an internal control with the primers BxACqF (TTC AGG TGT TAC CCA CAC CG) and BxACqR (GCG GTG GTG GTG AAA GAG TA). Each treatment was performed on three separate biological replicates, and each replicate was measured three times. The quantification and normalization of the data followed the $\log _{2}$ fold method according to the Promega instructions. Student's $t$-test was used to analyze differences between group grades. The second group was used to assess survival differences. The survival assay of $B$. xylophilus was performed in 24-well

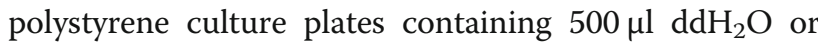
carvone $(800 \mathrm{mg} / \mathrm{L})$ per well in the dark at $25^{\circ} \mathrm{C}$. One thousand nematodes were then added to each well. Moving and immobilized nematodes were counted every 2 days. The third group was used to assess the pathogenicity difference of $P$. massoniana. RNAi-treated nematodes were inoculated into 3-year-old $P$. massoniana seedlings $(10,000$ Bx-cathepsin $W$ RNAi nematodes in $0.5 \mathrm{ml} \mathrm{ddH} \mathrm{H}_{2} \mathrm{O} /$ seedling) to test the pathogenicity of $P$. massoniana. The inoculations of M9 buffer-treated nematodes and $\mathrm{ddH}_{2} \mathrm{O}$ were set as the control. Thirty days after inoculation, nematodes were isolated and counted from $P$. massoniana seedlings. Three biological replicates were performed for the inoculation.

\section{Correlation of Bx-cathepsin $W$ to the susceptibility of pines}

Weighted gene coexpression network analysis (WGCNA) is a method frequently used to explore the complex relationships between genes and phenotypes [20]. The distinct advantage is that WGCNA transforms gene expression data into coexpression modules, providing insights into signaling networks that may be responsible for phenotypic traits of interest [21]. Therefore, in this study, we aimed to construct coexpression modules using the expression data of different pine-induced B. xylophilus genes. The module to which $B x$-cathepsin $W$ belonged and the association between its expression level and the susceptibility of different species of pines were identified. The survival rate of $B$. xylophilus and the disease susceptibility index of different species of pines (P. thunbergii $>$ P. massoniana $>$ P. sylvestris var. mongolica, our unpublished data) were selected as two traits for WGCNA.

Altogether, 6 transcriptomes (from nematodes induced by $P$. massoniana for 1 day and 15 days, $P$. thunbergii for 1 day and 15 days, $P$. sylvestris var. mongolica for 15 days, and CK2, a control of nematodes cultured on B. cinerea) 
were sequenced and analyzed according to the same method described above and were designated as the second sequencing. Two transcriptomes from the first sequencing (Treatment 1 and CK1) and all 6 transcriptomes from the second sequencing were analyzed by weighted gene WGCNA to find clusters (modules) of highly correlated anti-phytoalexin genes (Additional file 1: Text S5) [22]. The appropriate power value was determined when the degree of independence was over 0.8. The minimum number of genes was set as 30 for the high reliability of the results. For each expression profile, gene significance (GS) was calculated as the absolute value of the correlation between expression profile and each trait; module membership (MM) was defined as the correlation of expression profile and each module eigengene.

\section{Results}

Transcriptome sequencing and differentially expressed genes identification

To characterize the anti-phytoalexin gene transcript patterns, 4 cDNA libraries (Treatment 1 , CK1, Treatment 2, and CK2) of B. xylophilus were constructed and sequenced. The dataset was deposited in the SRA (Accession No. SRS3880513, NCBI Submission ID: SUB4555785, and BioProject ID: PRJNA494797).

An average of $79.69 \mathrm{Mb}$ raw reads was obtained for each sample. After removing the low-quality reads, more than 66.74 Mb clean reads (6.62 Gb clean bases) for each sample were obtained. Clean reads were mapped to the reference genome, the result showed that more than $93 \%$ of the transcripts could mapping to the reference genome (Additional file 1: Table S1) [9]. A total of 17,747 assembled unigenes were generated from the 4 libraries. On the basis of differential expression analysis [23] and the FDR [17], 2051 differentially expressed genes (including 784 significant differentially expressed genes) and 15,696 unchanged or nonsignificant differentially expressed genes were found from the Treatment 1 and CK1 libraries. Meanwhile, 1452 differentially expressed genes (including 715 significant differentially expressed genes) and 16,295 unchanged or nonsignificant differentially expressed genes were found from the Treatment 2 and CK2 libraries.

\section{Anti-phytoalexin genes identification}

A total of 2051 P. massoniana-induced genes were found, including 584 promoted and 1467 suppressed genes (Fig. 1a Treatment 1). Furthermore, 1452 carvoneinduced genes were identified, including 796 promoted and 656 suppressed genes (Fig. 1a Treatment 2). The results indicated that more genes responded to induction by $P$. massoniana than induction by carvone, which might be because $B$. xylophilus has to face a more complex mixture of compounds in the resin canals of pine hosts. Therefore, we filtered anti-phytoalexin genes by intersections of lists of $P$. massoniana-induced genes and carvone-induced genes. Altogether, 187 anti-phytoalexin genes were identified. Additionally, we identified 343 suppressed genes that showed the same expression patterns when induced by both Pinus massoniana and carvone (Fig. 1b), and the expression of those promoted and suppressed genes is detailed in Fig. 1c.

Further analysis showed that the 187 anti-phytoalexin genes could be divided into 4 groups. The 1st group included 29 pioneer genes, which had no homolog in either the NCBI Nonredundant sequences (nr) database or the genome of Caenorhabditis elegans. The 2nd group included 84 genes that had a homolog in the $\mathrm{nr}$ database (including 23 hypothetical proteins or uncharacterized proteins) but had no homolog in the genome of C. elegans. The 3rd group included 54 genes that had a homo$\log$ in the genome of $C$. elegans. The 4th group included 20 xenobiotic-metabolizing enzyme (XME) genes (Additional file 1: Table S2).

Among those 187 anti-phytoalexin genes, 95 genes could be enriched as 66 KEGG orthologs (Additional file 1: Table S3), 33 genes were enriched as Lysosome pathway especially (Additional file 1: Table S4), 4 antiphytoalexin genes were enriched as cathepsins (K01373 or K01365), which were in the pathways Lysosome (ko04142), Phagosome (ko04145), Antigen processing and presentation (ko04612), and Apoptosis (ko04210). Since cathepsins help to maintain metabolic homeostasis by participating in the degradation of heterophagic and autophagic material [24], homologous Cathepsin Wencoding genes were studied.

\section{Identification of homologous Cathepsin W-encoding genes}

A cDNA for a homolog of a cysteine protease, designated $B x$-cathepsin W (BUX.s00600.106), was cloned from the Treatment 2 cDNA library. Bx-cathepsin $W$ contained a coding sequence of $1179 \mathrm{bp}$, encoding a polypeptide of 392 amino acids composed of a putative 16-residue signal peptide, 141-residue propeptide, and a 235-residue mature protein. Protein sequence comparisons revealed $77 \%$ homology with human cathepsin W (E value =3e-43) [25], $76 \%$ homology with $C$. elegans cathepsin F (E value $=3 \mathrm{e}-$ 49), and approximately 53 75\% homology with human cathepsins $\mathrm{F}, \mathrm{O}, \mathrm{X}, \mathrm{K}, \mathrm{H}$, and $\mathrm{S}$ (E value $=3 \mathrm{e}-45 \sim 1 \mathrm{e}-39$ ). The deduced amino acid sequence contained the highly conserved residues of the catalytic triad of papain-like cysteine proteases (Fig. 2a). The results indicated that the protein belonged to the peptidase C1A subfamily (MEROPS database nomenclature), which was composed of cysteine peptidases that were similar to papain. The highly conserved residues of the catalytic triad (Cys197 and His340) formed a catalytic dyad (Fig. 2a and c). Two other residues played important roles in catalysis: a Gln191 
A
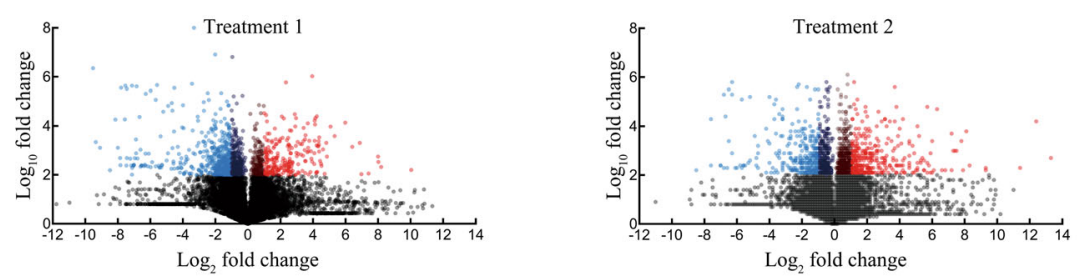

B
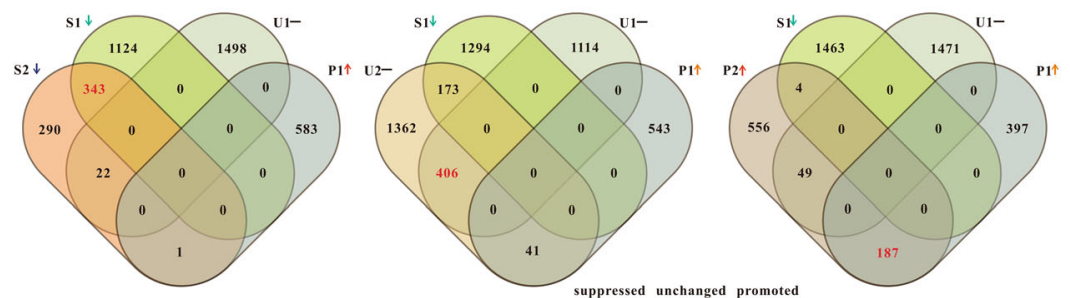

$\begin{array}{llll}\text { Treatment 1 } & \text { S1 } & \text { U1- } & \text { P11 } \\ \text { Treatment2 } & \text { S24 } & \text { U2- } & \text { P2 } 21\end{array}$

$\mathrm{C}$
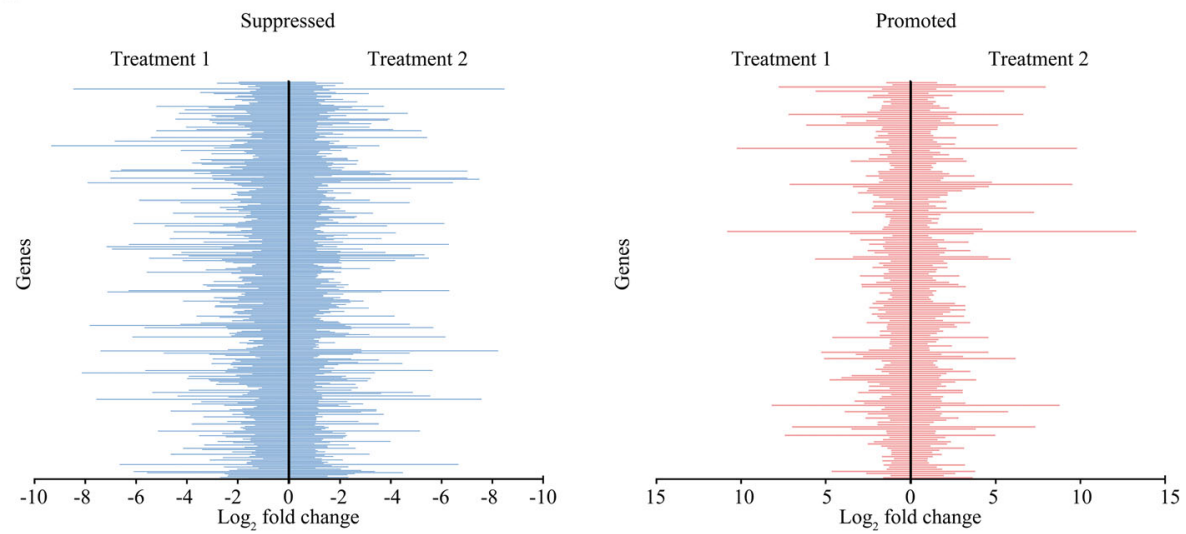

Fig. 1 Identification of carvone- and P. massoniana-induced genes (all figures were generated by the authors of this study). a. Scatter plot of the gene expression of $P$. massoniana-induced and carvone-induced genes in Treatment 1 and Treatment 2 libraries according to the log 2 fold change of normalized reads and the - $\log _{10}$ fold change of the $p$-value ( $t$-test, $\left.n=3\right)$. Expression levels are normalized by the mean numbers of transcripts per million clean reads (TPM). Data points colored blue, red or black represent suppressed genes, promoted genes or nonsignificant differentially expressed genes. $\mathbf{b}$. The intersections of lists of $P$. massoniana-induced and carvone-induced genes. The Venn diagram displays the distribution of 3571 unique genes from Treatment 1 and 3434 unique genes from Treatment 2 . S1 and S2 represent suppressed genes ( $\log _{2}$ fold $<-1, p<0.05$ and FDR $\left.<0.05\right)$. U1 and $U 2$ represent unchanged genes $\left(-1<\log _{2}\right.$ fold $<1$ or $p>$ 0.05). P1 and P2 represent promoted genes $\left(\log _{2}\right.$ fold $>1, p<0.05$ and FDR $\left.<0.05\right)$. c. Profiles of genes that show the same expression change trends when induced by both Pinus massoniana and carvone. Treatment 1: Induction of B. xylophilus gene expression by $P$. massoniana. Treatment 2: Induction of B. xylophilus gene expression by carvone

preceding the catalytic Cys197 and an Asn360 residue that orients the imidazolium ring of the catalytic His340 (Fig. $2 \mathrm{a}$ and $\mathrm{c}$ ). The crystal structure of Bx-cathepsin W was typical of C1A cysteine proteases. The $\mathrm{N}$-terminal propeptide contained $5 \alpha$-helices (Fig. $2 b \alpha 1 \sim \alpha 5$ ) and was in close contact with the mature domain, which comprised $5 \alpha$ helices and a $\beta$-sheet formed from 4 antiparallel $\beta$-strands.

\section{RNAi of Bx-cathepsin W}

A potent and specific $B x$-cathepsin $W$ silencing was found in $B$. xylophilus after the nematode soaked in siRNAs overnight. The patterns of fluorescein observed for the nematodes indicated that FAM/Cy3 labeled siRNA was taken up by B. xylophilus effectively (Fig. 3b and d). RTqPCR showed significant suppression of Bx-cathepsin $W$, whereas there was no mutual interference between the two genes of Bx-cathepsin $W$ and Bx-Actin after soaking in the relevant siRNA for $12 \mathrm{~h}$ (Fig. 3e). For the expression of $B x$-cathepsin $W$, no significant difference between the $B x$-Actin siRNA and nontargeting siRNA buffer was found after soaking for $12 \mathrm{~h}$. None of the RNAi-treated nematodes presented a significant difference in survival rate 
A PAPAIN PAPAIN
Cathepsin-W Bx-Cathepsin-W
Clustal Consensus

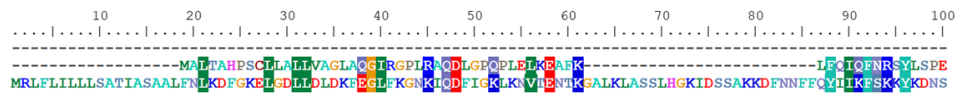
PAPAIN Cathepsin-W Clustal Consensus

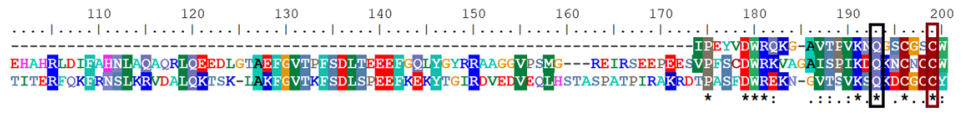

PAPATN

Cathepsin-W Bx-Cathepsin-w

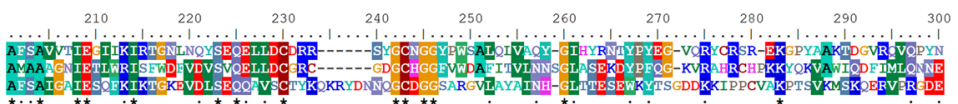

PAPAIN

Cathepsin-W

Bx-Cathepsin-W

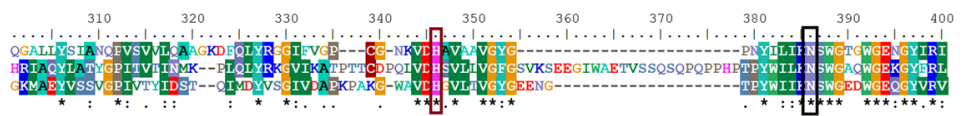

PAPAIN

Cathepsin-W Clustal Consensus

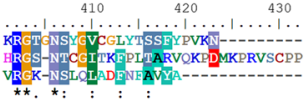

B
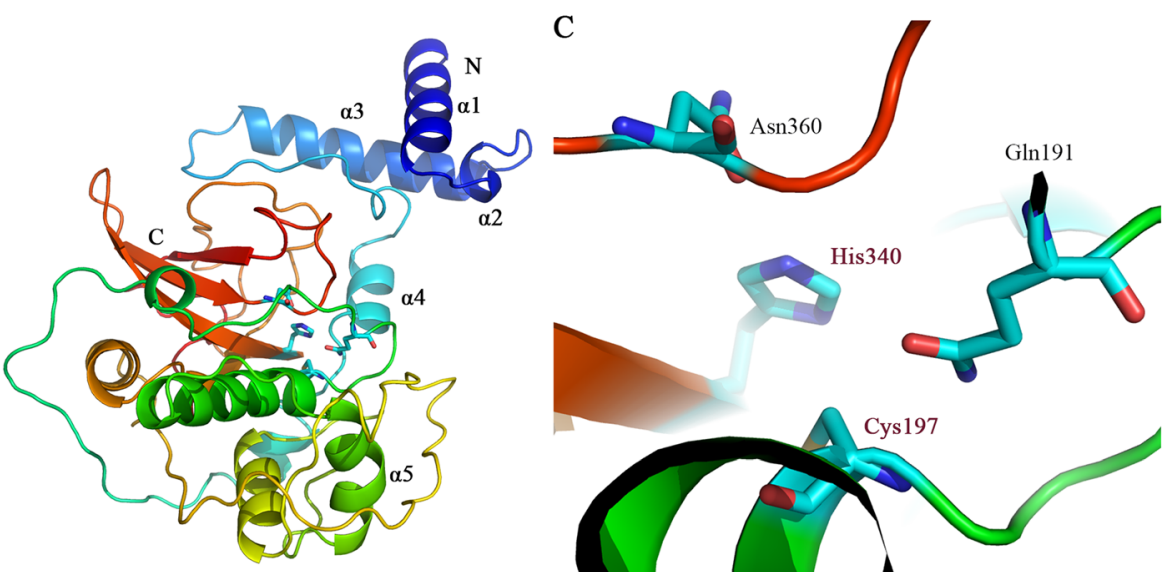

Fig. 2 Alignment and structure of Bx-cathepsin W (all figures were generated by the authors of this study). a. Multiple-sequence alignment of Bxcathepsin and homologs (papain and cathepsin W). Highly conserved residues of the catalytic triad are labeled as red boxes. The residues that play an important role in catalysis are labeled as black boxes. $\mathbf{b}$. The dimeric structure of Bx-cathepsin $\mathbf{W}$. The N-terminal a-helices are labeled a1 a5. c. The dimeric structure of the catalytic active site

(Student's $t$-test, $n=5$, bivariate correlation analysis, $B x$ cathepsin $W$ RNAi $p=0.68, B x$-Actin RNAi $p=1.00)$ and morphology (Fig. 3a and c) compared to the M9 buffertreated group during the whole 10-day study.

However, the survival rate of the $B x$-cathepsin $W$ RNAitreated nematode was reduced significantly after being soaked in the carvone solution compared to that of the M9 buffer-treated nematodes (Fig. 3f. Student's $t$-test, $\mathrm{n}=$ 5 , bivariate correlation analysis, $p=0.00$ ). This result indicated that the resistance of $B$. xylophilus to carvone decreased after the knockdown of $B x$-cathepsin $W$.

More importantly, significant pathogenicity attenuation in the Bx-cathepsin RNAi-treated nematodes was found (Fig. 4). Significant variations occurred in seedlings inoculated with Bx-cathepsin W RNAi-treated B. xylophilus (Fig. 4f) compared with the M9 buffer-treated nematodes (Fig. 4e). After 30 days, seedlings inoculated with the $B x$ cathepsin W RNAi-treated B. xylophilus were not completely wilted; in contrast, all seedlings inoculated with
M9 buffer-treated nematodes were completely wilted, while no wilt symptoms were found in the $\mathrm{ddH}_{2} \mathrm{O}$-inoculated seedlings. After 30 days, few nematodes could be isolated from the $B x$-cathepsin RNAi-treated nematode inoculation (Fig. 4c, f, and i). This result indicated that the knockdown of $B x$-cathepsin $W$ reduced the survival rates of B. xylophilus under P. massoniana stress.

\section{Correlation of Bx-cathepsin $W$ to the susceptibility of pines}

Based on the initial results, expression values of 17,624 genes in 8 transcriptomes of B. xylophilus were used to construct the coexpression module with WGCNA package tools. First, the power value was screened out (Fig. 5a). When the power value was equal to 12 , the independence degree was up to 0.82 , and the average connectivity degree was higher. Therefore, this power value was used to construct coexpression modules, and 25 distinct gene coexpression modules were identified in $B$. 

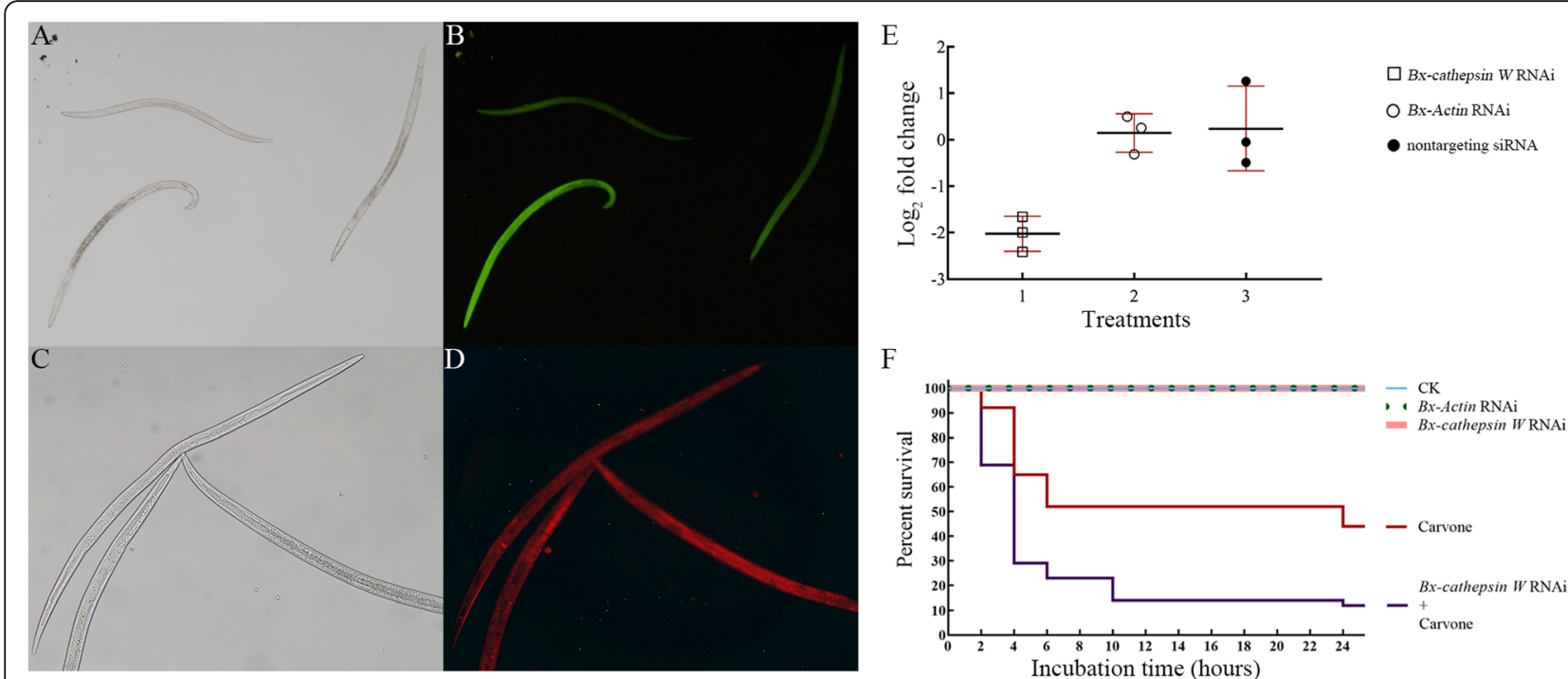

Fig. 3 Bx-cathepsin W RNAi in B. xylophilus (all figures were generated by the authors of this study). a. Morphology of Bx-cathepsin W RNAi-treated B. xylophilus. $\mathbf{b}$. The green fluorescence indicates the FAM-labeled Bx-cathepsin W corresponding with effective siRNA entry into the nematode. $\mathbf{c}$. Morphology of Bx-Actin RNAi-treated B. xylophilus. $\mathbf{d}$. The red fluorescence indicates the Cy3-labeled Bx-Actin corresponding with effective siRNA entry into the nematode. e. The expression of $B x$-cathepsin $W$ detected by RT-qPCR after soaking in the relevant siRNA of Bx-cathepsin W, Bx-Actin, and nontargeting. f. Percent survival of Bx-cathepsin W RNAi- and carvone-treated B. xylophilus

xylophilus. These coexpression modules were constructed and shown in different colors, and the number of genes in the 25 modules is shown in Additional file 1: Table S5 (Fig. 5b). Interactions of the 25 coexpression modules were analyzed (Fig. 5c). Modules with common expression pattern interaction analysis of coexpression modules that were associated with particular traits were identified based on the correlation between module eigengene and traits (Fig. 6a). Genes in the yellow module show different expression patterns for different time points. The expression of most genes increased when $B$. xylophilus was inoculated into pine trees for 1 day (Fig. $5 \mathrm{~d}$ bar 2 and 5) or 3 days (Fig. 5d bar 7), and few genes were promoted at 15 days (Fig. $5 \mathrm{~d}$ bar 3,4 and 6).

The survival rate of B. xylophilus and the disease susceptibility index of different species of pine were selected for the two traits. Interaction analysis of coexpression modules that were associated with particular traits were identified based on the correlation between module eigengene and trait (Fig. 6a). The module eigengene is defined as the first principal component of a given module. It can be considered a representative of the gene expression profiles in a module. The expression profile of the eigengene of the yellow module, to which $B x$-cathep$\sin W$ belongs, was positively correlated to the survival rate of the nematodes (correlation: 0.61 , and $p$-value: 0.08 ) and pine susceptibility (correlation: 0.7 , and $p$ value: 0.04$)$, which indicated that genes in this module were related to the interaction between nematodes and pines. The GS of $B x$-cathepsin $W$ with survival rate was
0.79 ( $p$-value: 0.01 ), the GS of $B x$-cathepsin $W$ with pine susceptibility was 0.77 ( $p$-value: 0.01 ), and the MM of $B x$-cathepsin $W$ with yellow module was 0.79 ( $p$-value: $0.01)$. This result indicated that $B x$-cathepsin $W$ had a strong positive correlation with the susceptibility of pines; in other words, $B x$-cathepsin $W$ could improve the anti-phytotoxin ability of $B$. xylophilus, which was consistent with the $B x$-cathepsin $W$ RNAi analysis and suggested that $B x$-cathepsin $W$ functions as an antiphytotoxin via an additional method of analysis.

Additionally, $661 B x$-cathepsin W-correlated genes were identified from the yellow module; among them, the connections of the corresponding topological overlap of 439 genes were above a threshold of 0.08 (Fig. 6b). Further analysis of these 439 genes indicated that there were $10 B x$-cathepsin $W$-correlated anti-phytoalexin genes (Additional file 1: Table S6). The Bx-cathepsin Wcorrelated anti-phytoalexin genes also had 4421 interactions with additional 812 genes.

\section{Discussion}

The stress response is an essential process for B. xylophilus survival. Genomics research has identified many $B$. xylophilus stress response-related genes, such as the glutathione S-transferase [26], uridine diphosphate glucuronosyl transferase, cytochrome $\mathrm{P} 450$, and abscisic acid transporter gene families [9]. In this study, we compared B. xylophilus transcriptomes with the B. xylophilus genome and $C$. elegans genome to identify stress response-related genes, whereas the result indicated that 


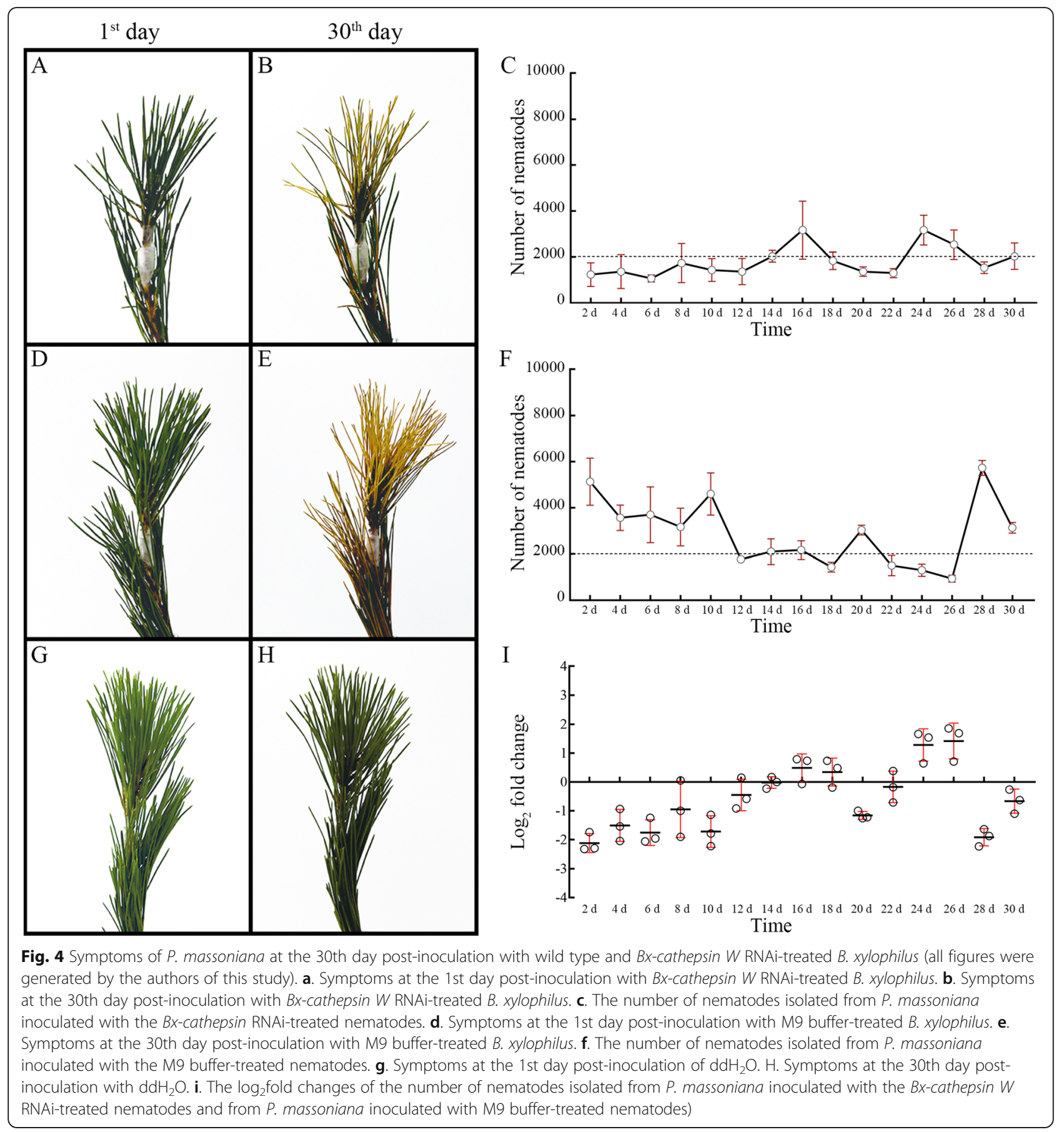

not all of these genes were stimulated (Additional file 1: Table S7) $[9,27,28]$. For example, only 68 cytochrome P450 monooxygenase genes and 35 xenobiotic transport across cell membranes related ATP-binding cassette transporter were found. This is consistent with previous transcriptomic studies about B. xylophilus parasitic adaptation to life on pine hosts, also only 48 cytochrome P450 family members were found [29]. Beyond this, 5 more UDP glucuronosyltransferases transcript were found, suggesting that there may be multiple splicing patterns. Previous transcriptome studies have also shown that only 12 genes were involved in the xenobiotic metabolism while the nematode transition from mycetophagous (fungal feeding) to phytophagous phase (nematodes infected to host) [30].

Furthermore, in order to find a common core among different phytoalexins induced B. xylophilus defense genes, all 187 anti-phytoalexin genes were compared 


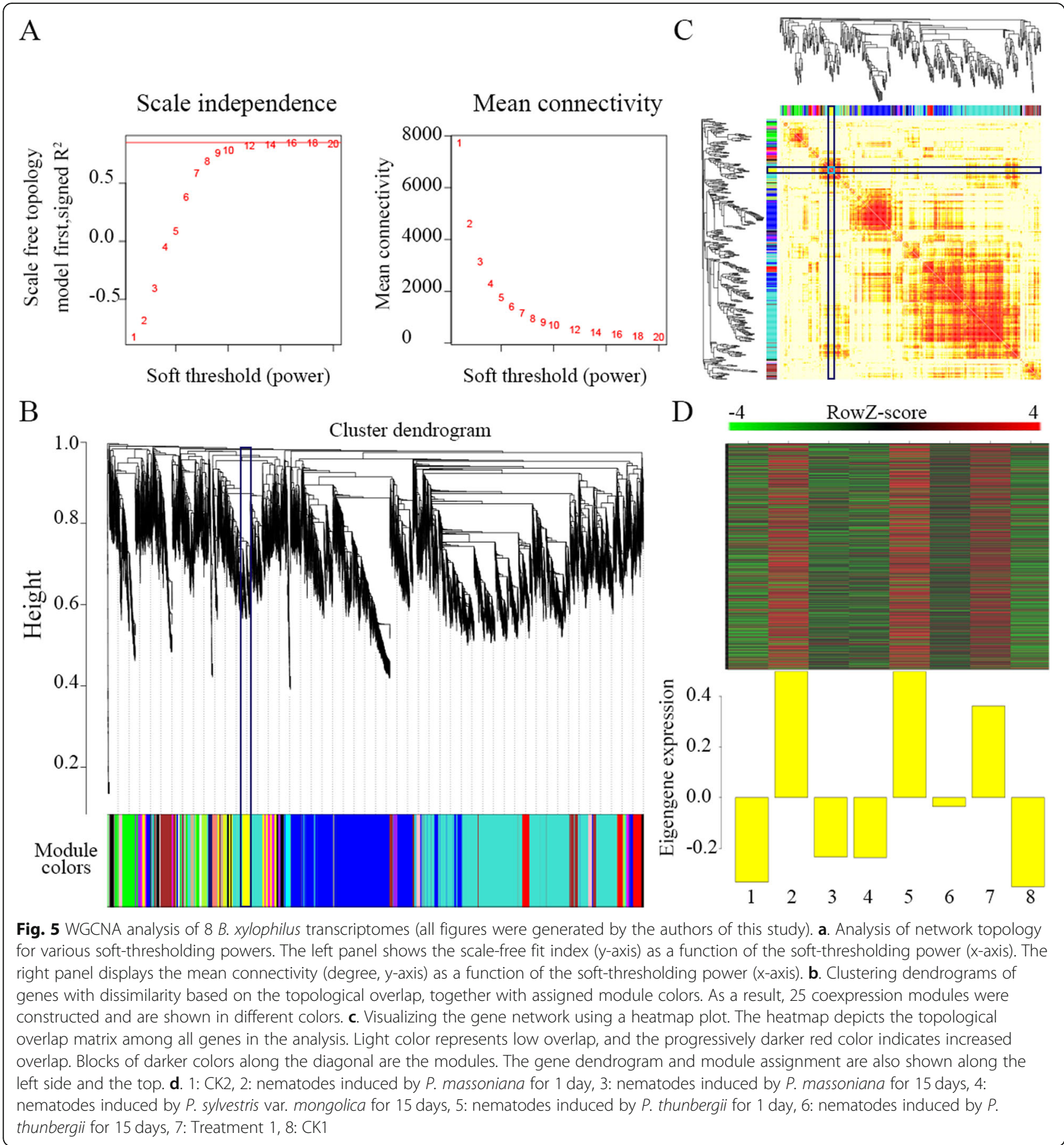

with other B. xylophilus transcriptome studies [31, 32]. However, only 2 genes (one small GTPase gene BUX.s01147.18 and one peroxidase gene BUX.c09083.1) were found to overlap with 41 DEGs induced by $\alpha$ pinene. And, only 1 gene (one peptidase gene BUX.s00422.693) were found to overlap with 30 DEGs between mycophagous and phytophagous stages B. xylophilus transcriptome. The DEGs between mycophagous and phytophagous reveal that B. xylophilus need a multilayer detoxification strategy in order to protect itself from host defense responses [32]. Those results indicate that maybe different phytoalexins induce nematode gene expression in its own way. Given the results, stress response-related genes may have more complex functions to allow B. xylophilus to cope with complex environments, such as interacting with other hosts, nematode-trapping fungus and other fungi (such as the blue-strain fungus) in the pine or the vector 

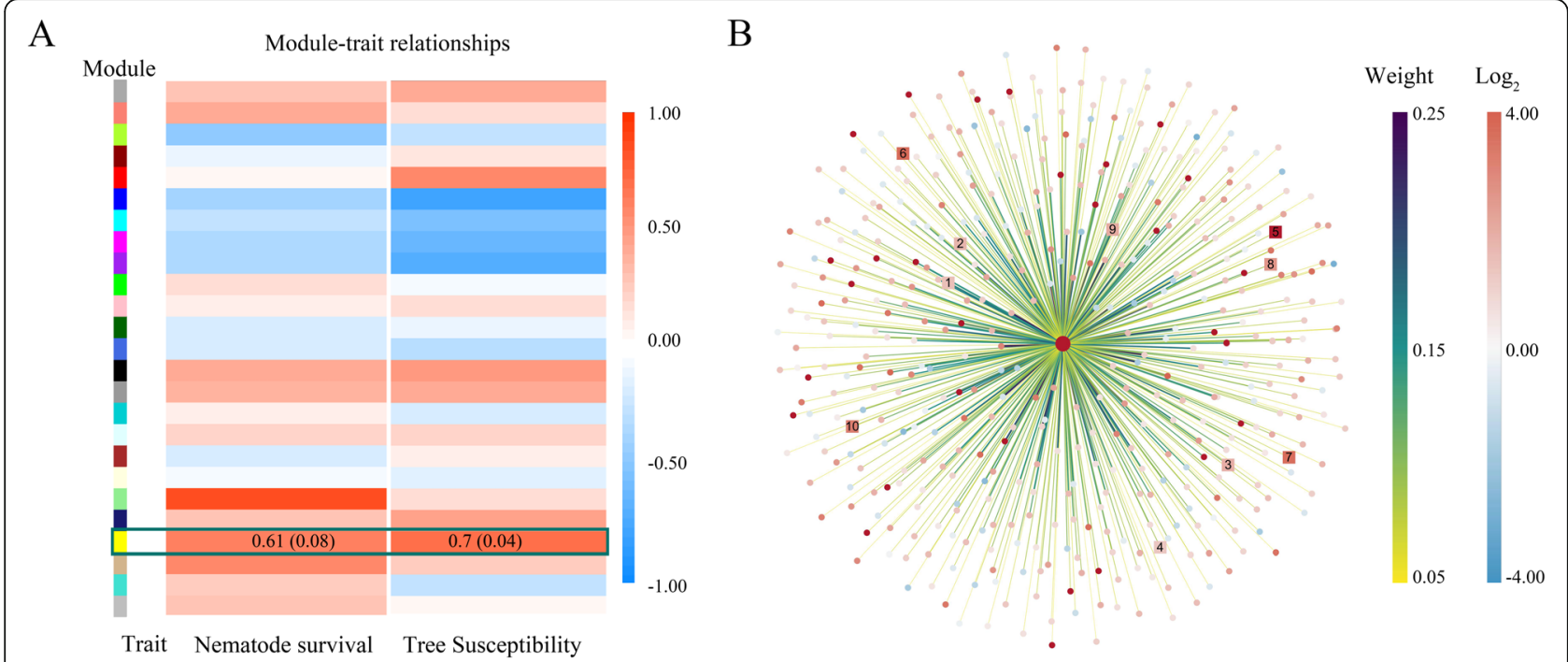

Fig. 6 Correlation of Bx-cathepsin $W$ to the susceptibility of pines and other genes in the yellow module (all figures were generated by the authors of this study). a. Module-trait associations. Each row corresponds to a module eigengene, and each column corresponds to a trait. The table is color-coded by correlation according to the color legend. b. Interaction of 439 Bx-cathepsin W-correlated genes identified from the yellow module. Each dot and square corresponds to a gene, with Bx-cathepsin $W$ at the center and the squares representing 10 anti-phytoalexin genes. The colors of the dots and squares represent the range of the gene's expression, $\log _{2}$ represent log 2 (Treatment 1/CK1)). The thickness and color of the lines represent connections of corresponding topological overlap

Monochamus alternatus. In particular, B. xylophilus must cope with phytoalexins, which are induced by pine in response to nematode infection. Antiphytoalexin is a crucial process for B. xylophilus to cope with its pine hosts.

In this study, we identified 187 anti-phytoalexin genes that showed the same promoted expression trends upon induction by both P. massoniana and carvone. However, the detailed functions of these anti-phytoalexin genes require further study. The roles of nematode cathepsins, one proteinase of cysteine proteinases (EC 3.4.22), may include invasion of host tissues, parasite nutrition and evasion of host defense responses [33, 34]. Cathepsins are secreted as inactive proenzymes and have an $\mathrm{N}$ terminal propeptide that is cleaved during maturation. Cathepsins L have been identified in the plant parasites Heterodera glycines [35] and Meloidogyne incognita [33]. Cysteine proteases are responsible for host hemoglobin degradation, as shown in Necator americanus [36] and Teladorsagia circumcincta [37]. Previous studies have suggested that cathepsins are involved in the digestive processes of adult $H$. glycines [35] and $M$. incognita [33] females. In addition, the function of the cysteine protease in $M$. incognita preparasitic infective $\mathrm{J} 2 \mathrm{~s}$ is directly related to the parasitic aspects of the plant-nematode interaction [33].

Therefore, we were particularly interested in determining whether $B x$-cathepsin $W$ functioned in the antiphytoalexin process of $B$. xylophilus in response to $P$. massoniana and carvone stresses. Carvone-treated $B$. xylophilus was not strictly correlated with feeding in Treatment 2, and the promotion of Bx-cathepsin $W$ expression indicates that some function of Bx-cathepsin $W$ is indeed more directly related to the plant-nematode interaction, especially to the anti-phytoalexin process. The results of the RNAi assessment indicated that $B x$ cathepsin $W$ supported the survival of $B$. xylophilus under carvone or $P$. massoniana stress. From the relationship between the expression level of Bx-cathepsin W and the survival rate of $B$. xylophilus in different pines, we found that the gene expression was positively related to the survival rate of $B$. xylophilus. Therefore, the correlation of the expression level of $B x$-cathepsin $W$ with the susceptibility of pines was examined. These results showed that Bx-cathepsin $W$ played an important role in improving the anti-phytotoxin ability of B. xylophilus. The survival rate of $B$. xylophilus was positively correlated with the susceptibility of pines; that is, the nematodes that infect more susceptible pines are more likely to survive and therefore are greater in number than nematodes that infect less susceptible pines. Therefore, based on the above results, $B x$-cathepsin $W$ is important in the pine wood nematode infection process. According to the correlation between genes in the yellow module, genes that shared the same expression pattern and had a high correlation with $B x$-cathepsin $W$ might have a strong regulatory relationship with $B x$-cathepsin $W$, which could indicate a direction for further research on Bx-cathepsin $W$ and a new target for the control of the nematode. 


\section{Conclusions}

The results indicated that the anti-phytoalexin genes, particularly $B x$-cathepsin $W$, support the survival of the nematode $B$. xylophilus under $P$. massoniana phytoalexin stress. The cDNA library sequencing, differentially expressed gene identification and WGCNA algorithm provided insight at a systemic level into the gene regulation of $B$. xylophilus in response to the immune reaction of $P$. massoniana. These results will lead to a better understanding of the function of nematode defenses in host innate immunity.

\section{Supplementary information}

Supplementary information accompanies this paper at https://doi.org/10. 1186/s12864-019-6167-2.

Additional file 1: Table S1. Statistical analysis of the RNA sequencing data. Table S2. Xenobiotic-metabolizing enzyme genes. Table S3. Results of KEGG enrichment. Table S4. Anti-phytoalexin genes related to the Lysosome pathway. Table S5. The number of genes in the 25 modules. Table S6. Bx-cathepsin W-correlated anti-phytoalexin genes. Table S7. Identification of B. xylophilus xenobiotic metabolism genes. Text S1. Transcriptome library preparation and sequencing. Text S2. Data analysis of reads. Text S3. Transcriptome assembly. Text S4. The differential gene expression analysis. Text S5. The WGCNA algorithm.

\section{Abbreviations}

Cy3: cyanine dyes 3; FAM: 5-carboxyfluorescein; FDR: false discovery rate; GS: gene significance; KEGG: Kyoto Encyclopedia of Genes and Genomes; MM: module membership; Nr: NCBI Nonredundant sequences database; RTqPCR: real-time quantitative PCR; SRA: Sequence Read Archive;

TPM: transcripts per million clean reads; Tween-20: polysorbate surfactant 20; WGCNA: weighted gene coexpression network analysis; XMEs: xenobioticmetabolizing enzymes

\section{Acknowledgements}

Not applicable.

\section{Authors' contributions}

Conceived and designed the experiments: FW, and DLL. Performed the experiments: QLC, YML, and FW. Analyzed the data: FW, QLC and DLL. Contributed reagents/materials/analytical tools: FW, DLL, QLC, YML, RQS, and RZZ. Wrote the paper: FW and QLC. All authors have read and approved the manuscript.

\section{Funding}

This work was supported by the National Key R \& D Program of China (2018YFC1200400), the National Natural Science Foundation of China (31971656, 31570637), and the Fundamental Research Funds for the Central Universities (2572017PZ08, 2572018AA04). The funders had no input into study design, data analysis, data interpretation or preparation of the manuscript.

\section{Availability of data and materials}

All supporting data are included as additional files as "Supplemental data files.docx".

\section{Ethics approval and consent to participate}

Not applicable.

\section{Consent for publication}

Not applicable.

\section{Competing interests}

The authors declare that they have no competing interest.
Received: 2 October 2018 Accepted: 2 October 2019

Published online: 26 October 2019

\section{References}

1. Jones JT, Moens M, Mota M, Li H, Kikuchi T. Bursaphelenchus xylophilus: opportunities in comparative genomics and molecular host-parasite interactions. Mol Plant Pathol. 2008:9(3):357-68.

2. Mota MBH, Bravo M, Penas A, Burgermeister W. First report of Bursaphelenchus xylophilus in Portugal and in Europe. Nematology. 1999;17:8.

3. Wang F, Wang Z, Li D, Chen Q. Identification and characterization of a Bursaphelenchus xylophilus (Aphelenchida: Aphelenchoididae) thermotolerance-related gene: BX-HSP90. Int J Mol Sci. 2012;13(7):8819-33.

4. Lapin D, Van den Ackerveken G. Susceptibility to plant disease: more than a failure of host immunity. Trends Plant Sci. 2013;18(10):546-54.

5. Hammerschmidt R. PHYTOALEXINS: what have we learned after 60 years? Annu Rev Ohytopathol. 1999;37:22.

6. Paxton JD. Phytoalexins - a working redefinition. J Phytopathol. 1981; 101(2):106-9.

7. Yamada T, Hanawa F, Nakashima T, Ito S: Phytoalexins accumulation in Pinus strobus following the pinewood nematode infection. In: Sustainability of pine forests in relation to pine wilt and decline Proceedings of International Symposium, Tokyo, Japan, 27-28 October, 1998: 1999; 1999.

8. Hanawa F, Yamada T, Nakashima T. Phytoalexins from Pinus strobus bark infected with pinewood nematode, bursaphelenchus xylophilus. Phytochemistry. 2001;57(2):223-8.

9. Kikuchi T, Cotton JA, Dalzell JJ, Hasegawa K, Kanzaki N, McVeigh P, Takanashi T, Tsai IJ, Assefa SA, Cock PJA, et al. Genomic insights into the origin of parasitism in the emerging plant pathogen Bursaphelenchus xylophilus. PLoS Pathog. 2011;7:9.

10. Mamiya Y, Shoji T. Pathogenicity of the pinewood nematode, Bursaphelenchus xylophilus, to Japanese larch, Larix kaempferi, seedlings. J Nematol. 2009:41(2):157-62.

11. Caboni P, Saba M, Tocco G, Casu L, Murgia A, Maxia A, Menkissoglu-Spiroudi $U$, Ntalli N. Nematicidal activity of mint aqueous extracts against the rootknot nematode Meloidogyne incognita. J Agric Food Chem. 2013;61(41): 9784-8.

12. Wang F, Li D, Wang Z, Dong A, Liu L, Wang B, Chen Q, Liu X. Transcriptomic analysis of the rice white tip nematode, Aphelenchoides besseyi (Nematoda: Aphelenchoididae). PLoS One. 2014;9(3):e91591.

13. Huang J, Liang X, Xuan Y, Geng C, Li Y, Lu H, Qu S, Mei X, Chen H, Yu T. A reference human genome dataset of the $B G I S E Q-500$ sequencer. Gigascience. 2017;6(5):1-9.

14. Zhu FY, Chen MX, Ye NH, Qiao WM, Gao B, Law WK, Tian Y, Zhang D, Zhang D, Liu TY. Comparative performance of the BGISEQ-500 and Illumina HiSeq4000 sequencing platforms for transcriptome analysis in plants. Plant Methods. 2018;14(1):69.

15. Kim D, Langmead B, Salzberg SL. HISAT: a fast spliced aligner with low memory requirements. Nat Methods. 2015;12(4):357.

16. Li D, Wang F, Wang C, Zou L, Wang Z, Chen Q, Niu C, Zhang R, Ling Y, Wang B. MicroRNA-mediated susceptible poplar gene expression regulation associated with the infection of virulent Melampsora larici-populina. BMC Genomics. 2016:17:59.

17. Benjamini $Y$, Yekutieli $D$. The control of the false discovery rate in multiple testing under dependency. Ann Stat. 2001;29:23.

18. Arnold K, Bordoli L, Schwede T. The SWISS-MODEL workspace: a web-based environment for protein structure homology modelling. Bioinformatics. 2006;22(2):195-201.

19. Alexander N, Woetzel N, Meiler J. bcl::cluster : a method for clustering biological molecules coupled with visualization in the Pymol molecular graphics system. IEEE Int Conf Comput Adv Bio Med Sci. 2011;2011:13-8.

20. Langfelder P, Horvath S. WGCNA: an R package for weighted correlation network analysis. BMC Bioinformatics. 2008;9:559.

21. Wan Q, Tang J, Han Y, Wang D. Co-expression modules construction by WGCNA and identify potential prognostic markers of uveal melanoma. Exp Eye Res. 2018;166:S0014483517303536.

22. Chen DJ, Qi L, Zhu J, Wan Z, Liu H, Min D, Guo R, Zhang B. Identifying osteosarcoma metastasis associated genes by weighted gene co-expression network analysis (WGCNA). Cellular Physiology \& Biochemistry International Journal of Experimental Cellular Physiology Biochemistry \& Pharmacology. 2018;45(5):1966. 
23. Anders $\mathrm{S}$, Huber $\mathrm{W}$. Differential expression analysis for sequence count data. Genome Biol. 2010;11(10):R106.

24. Repnik U, Stoka V, Turk V, Turk B. Lysosomes and lysosomal cathepsins in cell death. Biochim Biophys Acta. 2012;1824(1):22-33.

25. Linnevers C, Smeekens SP, Brömme D. Human cathepsin W, a putative cysteine protease predominantly expressed in CD8+ T-lymphocytes. FEBS Lett. 1997:405(3):253-9.

26. Espada M, Jones JT, Mota M. Characterization of glutathione S-transferases from the pine wood nematode, Bursaphelenchus xylophilus. Nematology. 2016;18:697.

27. Stein LD, Bao Z, Blasiar D, Blumenthal T, Brent MR, Chen N, Chinwalla A, Clarke L, Clee C, Coghlan A, et al. The genome sequence of Caenorhabditis briggsae: a platform for comparative genomics. PLoS Biol. 2003;1(2):E45.

28. Consortium CeS. Genome sequence of the nematode C. elegans: a platform for investigating biology. Science. 1998:282(5396):2012-8.

29. Yan X, Cheng XY, Wang YS, Luo J, Mao ZC, Ferris VR, Xie BY. Comparative transcriptomics of two pathogenic pinewood nematodes yields insights into parasitic adaptation to life on pine hosts. Gene. 2012;505(1):81-90.

30. Tsai IJ, Tanaka R, Kanzaki N, Akiba M, Yokoi T, Espada M, Jones JT, Kikuchi T. Transcriptional and morphological changes in the transition from mycetophagous to phytophagous phase in the plant-parasitic nematode Bursaphelenchus xylophilus. Mol Plant Pathol. 2016;17(1):77-83.

31. Li YMF, Deng $X$. Comparative transcriptome analysis of the pinewood nematode Bursaphelenchus xylophilus reveals the molecular mechanism underlying its defense response to host-derived a-pinene. International Journal of Molecular. 2019;20(4):16.

32. Espada M, Silva AC, Eves van den Akker S, Cock PJ, Mota M, Jones JT. Identification and characterization of parasitism genes from the pinewood nematode Bursaphelenchus xylophilus reveals a multilayered detoxification strategy. Mol Plant Pathol. 2016;17(2):286-95.

33. Neveu C, Abad P, Castagnonesereno P. Molecular cloning and characterization of an intestinal cathepsin $\mathrm{L}$ protease from the plant-parasitic nematode Meloidogyne incognita. Physiol Mol Plant Pathol. 2003;63(3):159-65.

34. Tort J, Brindley PJ, Knox D, Wolfe KH, Dalton JP. Proteinases and associated genes of parasitic helminths. Adv Parasitol. 1999;43(43):161.

35. Urwin PE, Lilley CJ, Mcpherson MJ, Atkinson HJ. Characterization of two CDNAs encoding cysteine proteinases from the soybean cyst nematode Heterodera glycines. Parasitology. 1997;114(6):605-13.

36. Brown A, Burleigh JM, Billett EE, Pritchard DI. An initial characterization of the proteolytic enzymes secreted by the adult stage of the human hookworm Necator americanus. Parasitology. 1995;110(5):555-63.

37. Young CJ, Mckeand JB, Knox DP. Proteinases released in vitro by the parasitic stages of Teladorsagia circumcincta, an ovine abomasal nematode. Parasitology. 1995;110(4):465-71.

\section{Publisher's Note}

Springer Nature remains neutral with regard to jurisdictional claims in published maps and institutional affiliations.

Ready to submit your research? Choose BMC and benefit from:

- fast, convenient online submission

- thorough peer review by experienced researchers in your field

- rapid publication on acceptance

- support for research data, including large and complex data types

- gold Open Access which fosters wider collaboration and increased citations

- maximum visibility for your research: over $100 \mathrm{M}$ website views per year

At BMC, research is always in progress.

Learn more biomedcentral.com/submissions 